\title{
The 8th Brazilian Congress of Social and Human Sciences in Health: continuity, convergence and coherence
}

| ${ }^{1}$ Martinho Silva I

${ }^{1}$ Instituto de Medicina Social, Universidade do Estado do Rio de Janeiro. Rio de Janeiro-RJ, Brazil (silmartinho@gmail.com). ORCID: 0000-0003-3577-958X

DOI: http://dx.doi.org/10.1590/S0103-73312019290400

About one thousand participants (CBCSHS..., 2019) walked approximately two thousand meters through the streets of the capital of a state in the Northeast of Brazil, in defense of more diversity and democracy in the Brazilian National Health System - SUS (EM PAUTA..., 2019). On a Saturday afternoon, September 28, 2019, almost half of the participants of the 8th Brazilian Congress of Social and Human Sciences in Health (CONGRESSO..., 2019) departed from the congress headquarters, the Federal University of Paraiba (UFPB), towards Praça da Paz, making the scientific event even more playful, cultural, artistic and political. The previous day, the Congress hosted a ceremony eagerly awaited by all: the celebration of the 40th anniversary of the Brazilian Association of Collective Health -Abrasco (ENCONTRO..., 2019). This mix of protest and commemoration marked the 8th Congress, held at UFPB, in João Pessoa, from September 26 to 30, 2019, on the theme "Equality in Differences: Confrontations in the joint construction of wellbeing and the SUS".

The 8th Brazilian Congress of Social and Human Sciences in Health had 2,168 participants, while the 7th Congress in Cuiabá, in 2016, received 958 (RELATÓRIO..., 2017); the 6th, in Rio de Janeiro in 2013, received 1,645; the 5th, in São Paulo in 2011, made it with 1,250; and the 1st, in Curitiba, in 1995 - when it was still called "Brazilian Congress of Social Sciences in Health", 400. 
The growth in the number of participants was accompanied by the increase in the number of higher education institutions that have been nominating representatives for the Abrasco Commission on Social and Human Sciences in Health, the event organizer. In 1995, only six institutions appointed representatives (CANESQUI, 1995). In 2011 this number increased to 10 (RELATÓRIO..., 2015) and in 2014 to 20 (RELATÓRIO..., 2016), reaching 29 in 2019 (COMPOSIÇÃO..., 2019). This expansion is the result of a collective effort, including professors from the Social Medicine Institute at Rio de Janeiro State University, such as Madel Luz, Luiz Antonio de Castro Santos, Kenneth R. Camargo Jr., Roseni Pinheiro, Francisco Ortega and Claudia Mora, who took turns as representatives of the Institute in the aforementioned Commission (PINHEIRO, 2013).

These congresses sometimes express the concentration of scientific production in the South-Southeast axis (IANNI et al., 2015), sometimes the regional capillarity of the Social and Human Sciences in Health (TRAD, 2012), so that one of the achievements of the 8 th Congress concerns precisely the fact that the vast majority of its thematic groups (WGs) - 27 of 29 - were multi-regional, ie most WGs were coordinated by people linked to institutions located in at least two of the five regions of Brazil, including North, Northeast and Midwest. The regional diversity of the WGs is in line with the very configuration of the Committee on Social and Human Health Sciences, unique to Abrasco's three committees - the other two being Epidemiology and Politics, Planning and Management - with representatives from universities in the five country regions. The capillarity, diversity and regional decentralization of the 8th Congress and the Committee represent a breakthrough in the fight against inequities in science and technology in Brazil, revealing the continuity of Committee management.

These and other achievements of the 8th Congress express, in addition to continuity and convergence, coherence between ideas and acts, as the teamwork of the national and local organizing committees, as well as the Congress scientific committee, enabled the combination of equality and difference at various levels. Among the innovations of the Congress, the following stand out: 1) students presented proposals of WGs, and some of WGs were coordinated by students of specialization courses; 2) members of social movements were part of the scientific committee, contributing to the construction of criteria for awarding the works presented in the 29 WGs; and 3) specific evaluation criteria were elaborated for the 
experience reports presented in these WGs, valuing the level of learning acquired in the experiences, as well as the exercise of critical thinking about the interventions.

None of this would have been possible without the protagonism of the local organizing committee in the whole process of building the Congress, composed by Eymard Vasconcelos, Pedro Cruz and other representatives of UFPB Popular Health Education, involved in extension projects that received national recognition (VASCONCELOS; CRUZ, 2013). The inhabitants of João Pessoa still suffer symbolic and material damage linked directly or indirectly to the national government scenario, such as regional prejudice (EXAME, 2019) and pollution on the beaches (PESQUISADORES..., 2019).

Approaching well-being (ACOSTA, 2016) becomes urgent when we live these and other social and environmental disasters, as this concept emerges as a critique of the idea of development: more than efficiency, we should talk about sufficiency, in a perspective focused on living together in community, which is distinct from welfare regarded from an exclusively individual point of view. Amid so much chauvinism and other forms of discrimination among the representatives of the current Federal Government, it is more than ever necessary to promote the debate on equality in differences in our congresses.

We brought together educators and students, poets and researchers, activists and representatives of indigenous peoples at João Pessoa's Praça da Paz during the 8th Congress, nourishing the hope that other public acts will happen in the events promoted by Abrasco and other Brazilian scientific associations that fight for human rights. We still have many confrontations ahead, as it is increasingly evident that the contemporary world is not at all friendly to a shared construction between academia, health services and community.

\section{References}

ACOSTA, A. O bem viver: uma oportunidade para imaginar outros mundos. Sáo Paulo: Autonomia Literária/Elefante, 2016.

CANESQUI, A. M. Apresentação. In: (Org.). Dilemas e desafios das Ciências Sociais na Saúde Coletiva. São Paulo: Hucitec-Abrasco; 1995. p. 11-18.

CBCSHS puxa uma primavera de luta em ato histórico. Abrasco, 2019. Disponível em: https:// www.abrasco.org.br/site/eventos/congresso-brasileiro-de-ciencias-sociais-e-humanas-em-saude/ cbcshs-puxa-uma-primavera-de-lutas-em-ato-historico/43034/. Acesso em: 27 out. 2019. 
COMPOSIÇÃO da Comissão de Ciências Sociais e Humanas em Saúde. Abrasco, 2019. Disponível em: https://www.abrasco.org.br/site/comissaodecienciassociaisehumanasemsaude/ composicao/. Acesso em: 27 out. 2019.

CONGRESSO Brasileiro de Ciências Sociais e Humanas em Saúde, 8. Abrasco, 2019. Disponível em: https://www.cshs.com.br/. Acesso em: 27 out. 2019.

EM PAUTA na Saúde. Canal Saúde, 2019. Disponível em: https://www.canalsaude.fiocruz.br/ canal/videoAberto/em-pauta-na-saude-04102019-epa-0143. Acesso em: 27 out. 2019.

ENCONTRO de gerações marca celebração dos 40 anos da Abrasco. Abrasco, 2019. Disponível em: https://www.abrasco.org.br/site/eventos/congresso-brasileiro-de-ciencias-sociais-ehumanas-em-saude/celebracao-40-anos-abrasco/42936/. Acesso em: 27 out. 2019.

EXAME. Bolsonaro: "Daqueles governadores de 'Paraíba', o pior é o do Maranhão". Revista Exame, 2019. Disponível em: https://exame.abril.com.br/brasil/bolsonaro-daquelesgovernadores-de-paraiba-o-pior-e-o-do-maranhao/. Acesso em: 27 out. 2019.

IANNI, A. M. Z. et al. Os Congressos Brasileiros de Ciências Sociais e Humanas em Saúde da Abrasco: um campo científico em disputa. Ciênc. saúde coletiva, Rio de Janeiro, v. 20, n. 2, p. 503513, fev. 2015. Disponível em: <http://www.scielo.br/scielo.php?script=sci_arttext\&pid=S141381232015000200503\&lng=en\&nrm=iso>. Acesso em: 27 out. 2019.

PESQUISADORES querem estado de emergência de saúde pública por óleo no Nordeste. $O$ Estado de São Paulo, 2019. Disponível em: https://sustentabilidade.estadao.com.br/noticias/ geral,pesquisadores-pedem-que-governo-declare-estado-de-emergencia-de-saude-publica-poroleo-no-nordeste,70003064788. Acesso em: 27 out. 2019.

PINHEIRO, R. Projeto Memória: 30 anos da Comissão de Ciências Sociais e Humanas em Saúde/Abrasco. Rio de Janeiro: UERJ/IMS/Lappis; 2013. Disponível em: https://www.abrasco. org.br/site/comissaodecienciassociaisehumanasemsaude/wp-content/uploads/sites/5/2016/11/ Projeto_Memoria_CBCSHS.pdf. Acesso em: 27 out. 2019.

RELATÓRIO de Gestão da Comissão de Ciências Sociais e Humanas em Saúde 20112015. Abrasco, 2015. Disponível em: http://www.abrasco.org.br/site/gtalimentacaoenutrica oemsaudecoletiva/wp-content/uploads/sites/18/2016/10/Relatorio-gest\%c3\%a3o-CCSHSabrasco-2011-2015.pdf. Acesso em: 27 out. 2019.

RELATÓRIO de Gestão da Comissão de Ciências Sociais e Humanas em Saúde 2014-2016. Abrasco, 2016. Disponível em: https://gestaoccshsabrasco.wordpress.com/composicao-2/. Acesso em: 27 out. 2019.

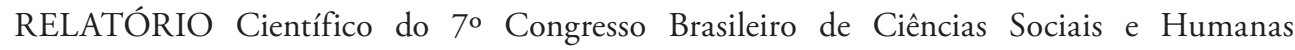
em Saúde. Abrasco, 2017. Disponível em: https:/www.abrasco.org.br/site/comissaodecie nciassociaisehumanasemsaude/wp-content/uploads/sites/5/2014/04/Relat\%C3\%B3rioCient\%C3\%ADfico_2017-04-18.pdf. Acesso em: 27 out. 2019. 
TRAD, L. A. B. Temas e enfoques contemporâneos nas Ciências Sociais e Humanas no Brasil: expressóes e tendências refletidas no V congresso da área. Cad. Saúde Pública. Rio de Janeiro, v. 28, n. 12, p. 2373-2379, 2012. Disponível em: http://www.scielo.br/scielo.php?script=sci_ arttext\&pid=S0102-311X2012001400016. Acesso em: 5 mar. 2019.

VASCONCELOS, E. M.; CRUZ, P. J. (Orgs.) Educação Popular na Formação Universitária: reflexões com base em uma experiência. São Paulo, João Pessoa: Hucitec, Editora Universitária da UFPB, 2013.

Finally, as usual in the editorial of the last issue of Physis in each year, we thank all the reviewers who helped us, from November / 2018 to October / 2019, in the difficult task of scientific publishing. Their names are listed below. Additionally, we would like to thank the most outstanding reviewer of the last 12 months for the quality and quantity of her collaborations: Leila Senna Maia, postdoctoral researcher and collaborating researcher at IMS-UERJ, who gave no less than eight opinions to our journal. To you, Leila, and all the other reviewers and authors, thank you very much!

\section{Reviewers - November 2018 / October 2019}

\begin{tabular}{|l|l|}
\hline Adelyne Mendes Pereira & Ana Raquel Lima \\
Adriana Greco & Anahí Farj \\
Adriano Maia dos Santos & André Felipe Cândido da Silva \\
Adriano Rosa & Andre Luis Bonifacio de Carvalho \\
Alessandro Campolina & André Luis de O. Mendonça \\
Alice Hirdes & André Rangel Rios \\
Amanda Ornela & Ândrea C. Souza \\
Ana Abraháo Silva & Andreza Nakano \\
Ana Carolina Feldenheimer & Bianca V. Sophia \\
Ana Claudia Monteiro & Camila Matias Pereira \\
Ana Cristina Figueiredo & Camilla Lopes Vieira \\
Ana Paula Guljor & Carine Blatt \\
Ana Paula Hemmi & Carinne Magnago \\
Ana Paula Melo & Carla Guedes \\
\hline
\end{tabular}


Carla Rocha Pereira

Carlos Dimas M. Ribeiro

Cecília Soares

Cinara Moutinho

Clarice Mota

Clarissa Seixas

Clarissa Tochetto

Claudia Cunha

Claudia Henschel de Lima

Claudia Jurberg

Claudia M. F. Penido

Claudia Passos Ferreira

Claudia Rezende

Cristiane Araújo

Cristiane Silvestre de Paula

Cristina Loyola

Charles Dalcanale Tesser

Christiana Profice

Daniel Groisman

Daniela Alves

Daniela Murta Amaral

Daniela Oliveira Melo

Daniela Ribeiro Schneider

Daniela Savi Geremia

Danilo Carvalho Oliveira

Deison Lucietto

Deivisson Vianna Santos

Denize Ribeiro

Edilson Marins Rodrigues Neto

Edna Aparecida Castro

Elaine Teixeira Rabello

Fabiana Pires

Fabio Pedro Balboa

Fatima Cecchetto

Felipe R. S. Machado

Fernanda Loureiro

Fernanda Martinhago

Fernanda Mendes
Flavia H. Freire

Frederico Policarpo

Gabriel Eduardo Schütz

Gaspar Maza

Gilberto Avalos

Grasiele Nespoli

Gustavo Figueiredo

Heloisa Morais

Hilderman Cardona

Hudson Pacífico

Iara Falleiros Braga

Isabela Samico

Islandia Maria Carvalho de Sousa

Izabel Passos

Jane Dutra Sayd

Jane M. Progianti

Jaqueline Ferreira

Joelson Rodrigues

Jorge Mathias do Nascimento

José Ricardo Ayres

Josué Laguardia

Julian Antman

Juliane Peruzzo

Júlio Simóes

Kalline Russo de Souza

Karen Matsumoto

Katia Aguiar

Kelly Alves

Kênia Silva

Laura López

Laura Murray

Laura Pozzana

Leandro Gonçalves

Leandro Wenceslau

Leila Senna Maia

Lenir Nascimento

Leny Alves Bomfim Trad

Liliane Carvalho 


\begin{tabular}{|c|c|}
\hline Lina Berardinelli & Maria Stella Castro Lobo \\
\hline Lucas Melo & Mariana Cruz \\
\hline Lucía Ariza & Mariana Pande \\
\hline Luciana Motta & Marilene Cabral do Nascimento \\
\hline Luciana Simas & Marina Guimarães Lima \\
\hline Luciana Surjus & Marina Nucci \\
\hline Luciane Miranda Guerra & Marisa Santos \\
\hline Luiz Augusto Souza & Marselle Carvalho \\
\hline Luiz Teixeira & Marta Rocha de Castro \\
\hline Luiza Costa & Marta Velloso \\
\hline Lumena Almeida Castro Furtado & Martinho Braga e Silva Batista \\
\hline Luna Rodrigues Freitas-Silva & Micheline Azevedo Meiners \\
\hline Luzia Oliveira & Michelle Cecille \\
\hline Magda S. Chagas & Mirna Teixeira \\
\hline Magda Scherer & Nébia Figueiredo \\
\hline Manoel Olavo Teixeira & Neide Emy Kurokawa e Silva \\
\hline Manuela Muller & Neli Maria Almeida \\
\hline Marcela Aguiar & Neuma Chaveiro \\
\hline Marcela Belardo & Nilia Prado \\
\hline Marcelo Mercante & Nivaldo Carneiro \\
\hline Marcia Fernanda de Mello Mendes & Nuria Malajovich \\
\hline Marcilio Dantas Brandão & Octavio Presgrave \\
\hline Marco Antônio Benamú & Pablo Rocon \\
\hline Marco Aurélio Jorge & Paloma Porto \\
\hline Marcos Nascimento & Patricia Almeida Fidelis \\
\hline Mardênia Pitombeira & Patrícia Araújo \\
\hline Mari Luz Esteban & Patricia Delfini \\
\hline Maria Belen Herrero & Paula Cerqueira \\
\hline Maria Cecília Carvalho & Paula Land \\
\hline Maria Claudia Veiga S. Carvalho & Paula Miura \\
\hline Maria Cristina Marcon & Paulo R. Fagundes \\
\hline Maria Cristina Ventura Couto & Pedro Barbosa \\
\hline Maria de Fátima Tavares & Pedro Paulo Gomes Pereira \\
\hline Maria Del Carmen Acuña & Petrônio José Martelli \\
\hline Maria Eduarda Moraes & Regina Facchini \\
\hline Maria Elizabeth Barros de Barros & Renarde Freire Nobre \\
\hline Maria Inês Moreira & Renata Vasconcelos \\
\hline Maria Luiza Cunha & Ricardo Burg Ceccim \\
\hline
\end{tabular}




\begin{tabular}{l|l} 
Ricardo Kuchenbecker & Simone Triquez \\
Ricardo Lugon & Sirleine Brandão de Souza \\
Roberta Romagnoli & Sônia Nicoletto \\
Roberto Amorim de Medeiros & Tânia Ferreira \\
Rodrigo Toniol & Tatiana Tavares da Silva \\
Roger Keller Celeste & Telma Menicucci \\
Ronaldo Teodoro dos Santos & Thaíse Bezerra \\
Rosa Rocha & Tiago Pires Marques \\
Rosana R.C. Castro & Valeska Zanello \\
Roseni Pinheiro & Vanessa Maia Rangel \\
Rosilda Mendes & Vanessa Silva \\
Rosilene Gomes & Vera Lúcia Mendes \\
Ruben Araujo de Mattos & Veronica Alcoforado \\
Sabrina Calil-Elias & Vladimir Araujo da Silva \\
Sandra Caponi & Wagner X. Camargo \\
Sandra M. Sales Fagundes & Walter Belik \\
Sérgio Alarcon & Xavier Medina \\
Shirley de Souza Simeão & Ximena Bermudez \\
Silvia Aguião & Yolanda Bodoque \\
\hline
\end{tabular}

